\title{
Polarimetric Imaging for Quality Control in Injection Molding
}

\author{
P. Nagorny $* a$, T. Lacombe $* a$, T. Muller $* a$, H. Favreliere ${ }^{a}$, E. Pairel ${ }^{a}$, M. Pillet $^{a}$ \\ ${ }^{a}$ Université Savoie Mont Blanc, SYMME, Annecy, France
}

\begin{abstract}
Quality control is a key requirement of the Fourth Industrial Revolution, especially in manufacturing. For high value technical parts, geometrical and appearance quality need to be guaranteed. The quality control of the part, directly on the manufacturing line, can be achieved with non-contact sensors. With a robust quality sensor, the manufacturing process could be adjusted in real time to always achieve the optimal quality. For quality control, visible spectrum imaging is commonly used. However, more information could be obtained from broader spectrum imaging and also from the degree of linear polarization of the light from the part. We have developed a simple polarimetric imaging system to verify whether linear polarization is capable of amplifying geometric and appearance defects. We evaluate the use of this polarimetric imager given the industrial constraints of injection molding. We compare supervised classification performances on an injection molded parts dataset, using polarimetry and non-polarized images. We compare the performances of different machine learning pipelines with hand-crafted feature extraction and Deep Transfer Learning. With its industrial robustness, polarimetry could be a valuable addition to non-contact imagers for geometric and appearance quality control.
\end{abstract}

Keywords: Polarimetry, Quality control, Injection Molding, Deep Learning, Discrete Modal Decomposition.

\section{INTRODUCTION}

Thermoplastics injection molding can be used to manufacture high value and complex parts in large quantities. To achieve the final product functionality, quality requirements often impose a control on every manufactured part. This control is done days after part production, sometime after other end processes like painting. If a defect appears, all the batch is rejected and in the case of a defect caused by the injection molding process, the painting time is also lost. A quality sensor which measure appearance and geometric quality just after molding could avoid this kind of defect. Moreover, measuring the quality of the part immediately after manufacture could allow the injection molding machine to be adjusted part by part in a closed-loop way to ensure that the best quality is always achieved. Requirements for an online quality sensor are the compatibility with the injection cycle time (ten to thirty seconds) and the integration and maintenance simplicity. A manufacturing line must be flexible. Multiple parts are produced on the same line. The quality sensor is only needed in case of highquality requirements. The geometrical quality of molded parts is difficult to control with contact-based system because of the elasticity of the material. It can be inferred with computer vision. The easiest defect to detect is the miss of a part (holes or not closed surfaces). For sub-millimeters defects, structured-light scanner or laser triangulation profilometers achieve good performances. In injection molding, a just-produced part is hot, and its geometry is not stabilized. For thick parts the cooling stabilization time can be a day. In previous works, we show that thermal imagery on hot parts can be used to infer the final cooled part geometry $1[2]$ However, appearance defects can sometimes be caused by geometric differences of the tens of micrometers order. To achieve micrometers resolution, confocal microscopy is needed, which has a critical part positioning requirement (sub-millimeter) and a high cost. The appearance quality can be measured using reflectance domes with multipoints of lighting system $\sqrt[3]{4}$ Those systems are able to detect micrometrics defects and are robust to the shift of the part positioning. However, they are difficult to integrate on manufacturing lines because of their size. A mono-point lighting system is simpler, but it requires precise positioning of the light equipment and sensor. Finally, it is not robust to the positioning shift of the measured part. It requires a precise part positioning which increase engineering complexity of the manufacturing line. In this paper, we propose to investigate the use of polarimetry as a simpler way to integrate sensor for a visual quality control task. Polarimetric imaging is commonly used with polymers to view mechanical stress using the stress-induced birefringence (photo-elasticity)

\footnotetext{
* Note an equal contribution. Correspondence: pierre.nagorny@univ-smb.fr
} 


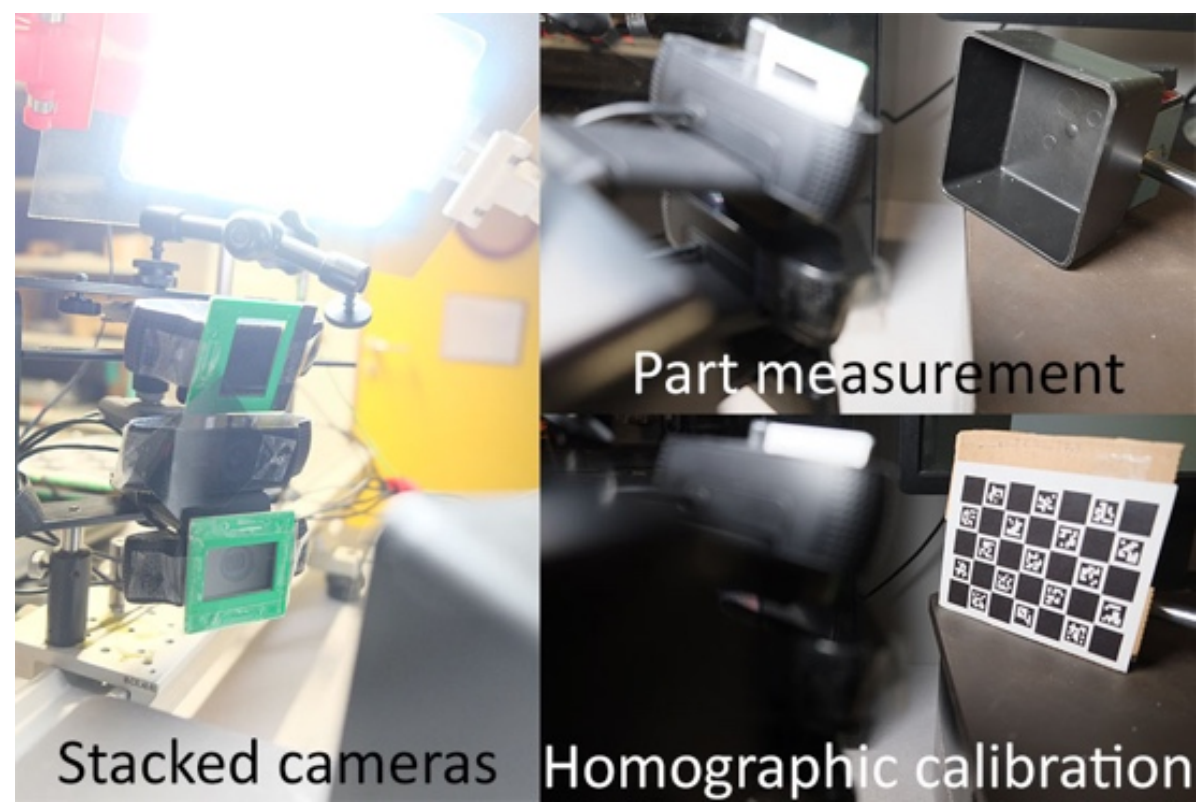

Figure 1: The polarimetric imager overview and calibration process.

on cooled transparent plastics materials .5 The measurement of the degree of linear polarization exists in many domains but the literature is very limited for the detection of appearances and geometric defects on plastic molded parts. The goal of this study is to explore polarimetric image processing from a new proposed sensor, using some machine learning tools. Traditional machine learning with hand-crafted feature extraction and more recent deep learning approaches are tested.

\section{METHODS}

In this part, we describe the polarimetric sensor we developed and used to acquire the images. It is followed by a short presentation of the methods used to create our database and to process it.

\subsection{A simple polarimetric imager}

Polarimetry imagers can be built with a unique imaging sensor and polarized filters directly integrated on the sensor plane (like in the recent Sony IMX250-MZR sensor). It limits optical complexity but it also limits the final image resolution. Other methods use a high-speed camera and a synchronized rotating linearly polarized filter, which adds mechanical complexity and limits the framerate. Finally, other sensors are made of multiple cameras stacked with a custom optical path designed to compensate the parallax difference ${ }^{6}$ Mirrors are commonly used to avoid the parallax between two cameras. When using three cameras or more, beam splitters, mirrors and prisms are needed. We choose a non-optical-based system to simplify the build. Our sensor relies on the fusion of three cameras polarized by linear filters at $0^{\circ}, 45^{\circ}$ and $90^{\circ}$ (Figure 1). To compensate the parallax difference between the three stacked cameras, we use the OpenCV library $y^{7}$ to compute an homographic transformation, which is calibrated on a ChAruco markers reference board 10 Parallax is compensated on a unique plane, thus only planar parts can actually be viewed in false colors. The parallax effect limits the human readability of our sensor if the object is not on the calibrated plane. More advanced pre-processing are discussed in Sec. 4.3. The polarimetric fusion software is open-sourced and available online.

However, in this study we do not use this planar homographic correction as we analyse directly raw images (Figure 2) using Machine Learning methods. The parallax difference in our system could also later be exploited for geometric measurements. We choose to limit ourselves to the minimal number of polarized images necessary to enhance defect detection. Three linearly polarized images at $0^{\circ}$ (I0), $45^{\circ}$ (I45) and $90^{\circ}$ (I90) are used for the polarized State Analyzer, and one linearly polarized light source at $0^{\circ}$ for the polarized State Generator. Those 
polarization angles are enough to recover the first third Stokes parameters, but not the full Stokes matrix as circular polarization is not measured. The Stokes equation 111 is:

$$
I(\theta, \Phi)=\frac{1}{2}\left[S_{0}+S_{1} \cos 2 \theta+S_{2} \cos \Phi \sin 2 \theta+S_{2} \sin \Phi \sin 2 \theta\right]
$$

We measure light intensity for three linear polarization angles $\theta$, but we do not measure the circular polarization angle $\Phi$ which we arbitrary define to zero:

$$
\begin{aligned}
I\left(0^{\circ}, 0^{\circ}\right) & =\frac{1}{2}\left[S_{0}+S_{1}\right] \\
I\left(45^{\circ}, 0^{\circ}\right) & =\frac{1}{2}\left[S_{0}+S_{2}\right] \\
I\left(90^{\circ}, 0^{\circ}\right) & =\frac{1}{2}\left[S_{0}-S_{1}\right]
\end{aligned}
$$

We get the partial Stokes vector!12

$$
S_{\text {partial }}=\left(\begin{array}{c}
S_{0} \\
S_{1} \\
S_{2}
\end{array}\right)=\left(\begin{array}{c}
I \\
Q \\
U
\end{array}\right)=\left(\begin{array}{c}
I\left(0^{\circ}, 0^{\circ}\right)+I\left(90^{\circ}, 0^{\circ}\right) \\
I\left(0^{\circ}, 0^{\circ}\right)-I\left(90^{\circ}, 0^{\circ}\right) \\
2 I\left(45^{\circ}, 0^{\circ}\right)-I\left(0^{\circ}, 0^{\circ}\right)-I\left(90^{\circ}, 0^{\circ}\right)
\end{array}\right)
$$

\subsection{Dataset creation}

Our industrial application case concerns the detection of defects in thermoplastic injection molding. Three main defects appear: stain, flow and jetting. An industrial production of 192 black boxes $(10 \mathrm{~cm} \times 10 \mathrm{~cm})$ has been run. The injection machine parameters are changed with twelve different configurations to produce multiple defects and degrees of defect, following a Design Of Experiment to explore the 12 machine parameters. Based on three experts quality evaluation, parts are sorted depending on the three main defects. A scale is set from 1 to 20 to evaluate the visual quality. In the present work, we choose to stay with a binary problem. Parts are labelled compliant if they are graded from 1 to 3 and non-compliant otherwise. In the dataset, images are reduced from $1920 \times 1200$ pixels to $331 \times 331$ pixels due to computational limits. The full resolution images could later be exploited using a more powerful server. To evaluate the interest of polarization, we measure each part three time:

- Without polarized lighting, nor polarized filters on cameras (dataset_bench)

- Without polarized lighting, but with polarized filters on cameras (dataset_nopola)

- With polarized lighting and with polarized filters on cameras (dataset_pola)

With each dataset, we evaluate several Machine Learning pipelines performances in Sec. 3 . The question to be answered is: does the use of polarized images improve the part quality classification performances?

\subsection{Hand-crafted feature extraction}

To answer to the question in Sec. 2.2, selected hand-crafted features are extracted from raw images to feed a classifier.

\subsubsection{Cooccurrence matrix}

The cooccurrence matrix, classified as a statistical feature extraction method, is used to characterize the texture of an image based on its pixel values distribution. The matrix is computed by counting all pairs of pixels with gray levels $i$ and $j$ separated by a distance $d$ in a given direction $\theta$. The matrix is often calculated by accumulating the values of cooccurrences in several directions to obtain a rotation invariant description. In this study, the matrices are calculated with $\theta=\left\{0, \pi, \frac{\pi}{2}, \frac{3 \pi}{4}\right\}$ and $d=1$. Once calculated, the cooccurrence matrices are usually reduced by extracting a set of features. The most popular ones have been proposed by Haralick $\frac{13}{13}$ and have been widely used in texture analysis. We used his 14 features. 

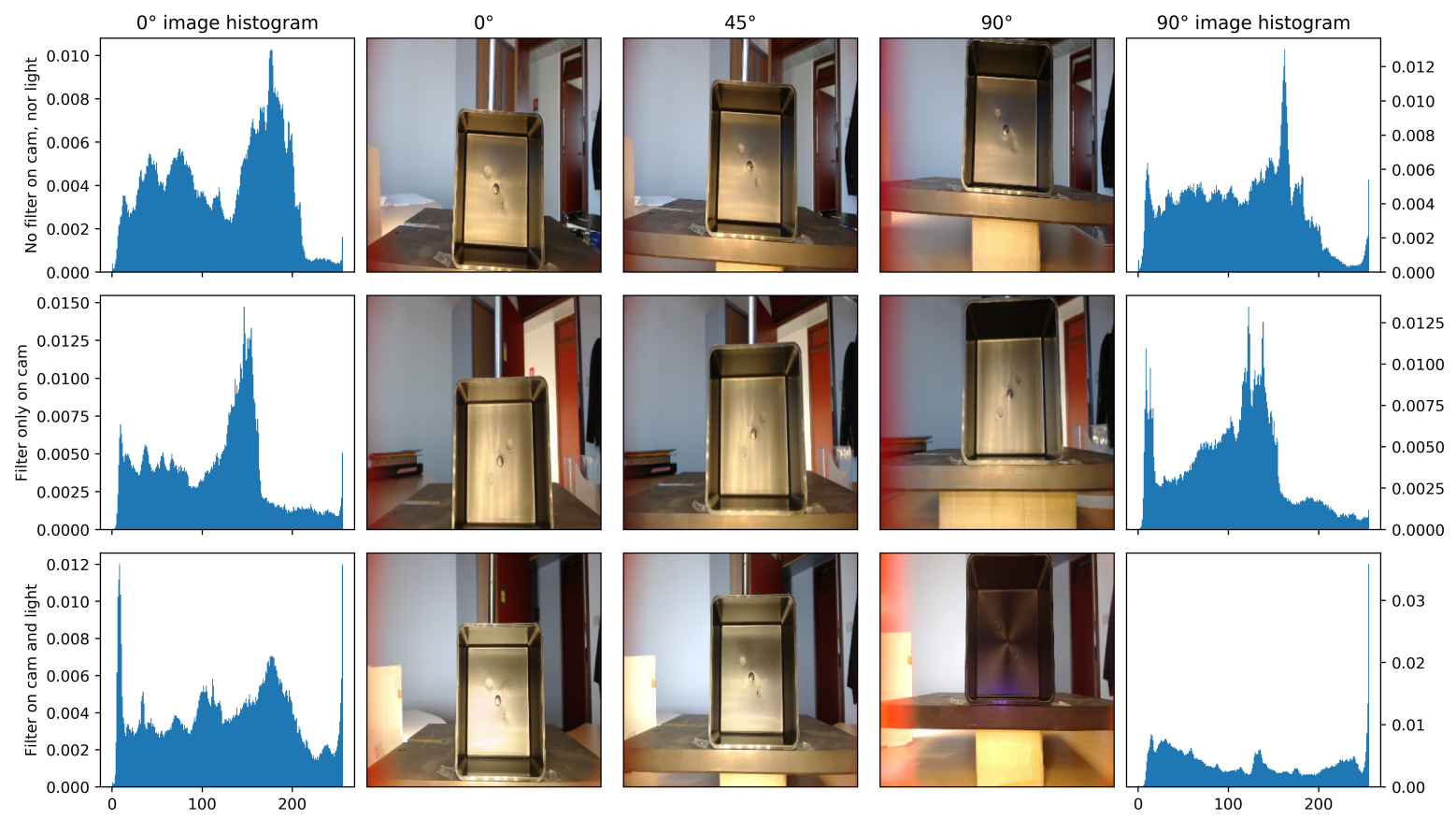

Figure 2: The same part in three datasets: raw images polarized or not.

\subsubsection{Discrete Modal Decomposition}

With the initial goal of automatically generating a 3D model for Computer Aided Design, Pentland ${ }^{\sqrt{14}}$ chose to use dynamic behavior of the object, i.e. its natural modes of vibration to describe its shape. Based upon this idea, the Discrete Modal Decomposition consists in decomposing a signal within a spectral basis built from eigen modes ${ }^{15}$ Similarly to the Discrete Fourier Transform or the Discrete Cosine Transform, this projection method allows to make the projection of the signal into an eigen basis built from structural dynamics. This eigen basis is defined by its eigen vectors, called modal vectors. The decomposition of the image pixel values in this set of modal vectors, called modal basis, produces a set of modal coordinates. These coordinates are used as features to analyse our images. For this study, we choose to use the first 100 coordinates deriving from this decomposition.

\subsection{Deep Transfer Learning}

Recent successes use Deep Learning model for image classification. The strength of Deep Neural Networks models is to not have to design any image preprocessing pipeline. First layers of the Deep Neural Networks take care of raw images preprocessing, then next layers do the feature extractions and selection. The use of convolutional networks for defect classification was done in Masci et al ${ }^{[16}$ on steel part. Further work was done on by Wen et al $\frac{17}{17}$ which evaluates the use of Convolutional Networks for bearing roller inspection. We evaluate different Convolutional Neural Networks ${ }^{\sqrt{18}}$ based classifiers using the Keras framework. ${ }^{[19}$ Best scores are obtained using Transfer Learning. Transfer Learning can be useful with small dataset, which is often the case with experimental practical works with an industrial frame. The method consists in replacing the last specialized layers of the network pre-trained on a large dataset with randomly initialized weights layers. These layers are then trained with the new dataset. Fine-tuning can be done by training the whole network for some epochs with the newly trained last layers. Our dataset is too small to train a network from scratch, even with data augmentation, which we used on all models. A previous work by Kim et al ${ }^{20}$ introduces a pre-trained VGG16 network ${ }^{21}$ on ImageNet, then fine-tuned on the DAGM 2007 dataset. ${ }^{22}$ We evaluate two pre-trained networks: VGG16 and MobileNetV2 ${ }^{23}$ The best performances in all cases are obtained with MobileNetV2: it has less parameters, it is faster to train and it performs well on our small dataset. It could also easily be embedded into low power inference system to perform defect classification directly on the production line. 


\subsection{Automated Machine Learning}

In addition of Deep tranfer Learning, we used different Automated Machine Learning (AutoML) with hyperparameters tuning to optimize ours Machine Learning pipelines. We found that AutoML is promising but it is still computationally costly. This constraint has to be taken into account to allow online models to train in a manufacturing environment. The TPOT library $24 \sqrt[25]{25}$ has been used to search an optimal Machine Learning model by genetic programming and the combination of multiple models with ensemble methods. ${ }^{26}$ The process tries multiple pipelines handling features selection and hyperparameters optimization. One single TPOT runs the evaluation of around 400 models in about 15 hours on the full $331 \times 331$ pixels images. AutoML on Deep Learning models is even more computational heavy and we did not evaluate this topic. Four hundred Machine Learning pipeline evaluations have been run over a month, using the Sacred framework ${ }^{27}$ to monitor live performances and to fix crashes faster.

\section{RESULTS}

In this section, we present classification scores on our Good/Bad parts problem for each Machine Learning pipelines.

\subsection{Hand-crafted feature extraction}

In images classification tasks with hand-crafted features, Support Vector Machines and Random Forests are commonly used as classifiers. In this study, the best scores are obtained through a SVM tuned with a Radial Basis Function kernel and a Cost parameter equal to 1. The score are obtained through a cross-validation process using a shuffle split with $30 \%$ of the dataset for the test, and 15 iterations in total. We use a $f 1$ evaluation score as we are dealing with a binary problem. The SciKit-Learn framework ${ }^{[28}$ was used. In Tables 1 and 2 , we compare performances with Haralick or Modal features from the 3 datasets presented in Sec. 2.2. The SVM classifier is used on the concatenation of the three images $\left(0^{\circ}, 45^{\circ}\right.$ and $\left.90^{\circ}\right)$ and separately on the $0^{\circ}$, on the $45^{\circ}$, and on the $90^{\circ}$ linearly polarized images. Scores are the mean and the standard deviation of the cross-validation analysis.

Table 1: Haralick features classification scores.

\begin{tabular}{|c|c|c|c|}
\hline Angle & dataset_pola & dataset_nopola & dataset_bench \\
\hline 3 angles & $0.90(+/-0.05)$ & $0.90(+/-0.04)$ & $0.90(+/-0.04)$ \\
$0^{\circ}$ & $0.90(+/-0.05)$ & $0.90(+/-0.04)$ & $0.90(+/-0.04)$ \\
$45^{\circ}$ & $0.90(+/-0.05)$ & $0.90(+/-0.04)$ & $0.90(+/-0.04)$ \\
$90^{\circ}$ & $0.90(+/-0.05)$ & $0.90(+/-0.04)$ & $0.90(+/-0.04)$ \\
\hline
\end{tabular}

Table 2: Modal features classification scores.

\begin{tabular}{|c|c|c|c|}
\hline Angle & dataset_pola & dataset_nopola & dataset_bench \\
\hline 3 angles & $0.90(+/-0.05)$ & $0.92(+/-0.05)$ & $0.92(+/-0.05)$ \\
$0^{\circ}$ & $0.90(+/-0.05)$ & $0.91(+/-0.05)$ & $0.92(+/-0.05)$ \\
$45^{\circ}$ & $\mathbf{0 . 9 3}(+/-\mathbf{0 . 0 5})$ & $0.93(+/-0.05)$ & $0.93(+/-0.05)$ \\
$90^{\circ}$ & $0.91(+/-0.06)$ & $0.92(+/-0.05)$ & $0.93(+/-0.05)$ \\
\hline
\end{tabular}

\subsection{Deep Transfer Learning}

Using the same formalism, the classification scores with a classifier made of a fine-tuned MobileNetV2 networks are shown in Tab. 3 . Due to computation time constraints, we do not cross-validate this classifier with shufflesplit but we keep the same training and validation set. The dataset is split with eighty percent (153 parts) in training set and twenty percent (39 parts) to validate performances. 
Table 3: Transfer Learning classification scores.

\begin{tabular}{|c|c|c|c|}
\hline Angle & dataset_pola & dataset_nopola & dataset_bench \\
\hline 3 angles & 0.781 & 0.781 & 0.781 \\
$0^{\circ}$ & 0.719 & 0.719 & $\mathbf{0 . 9 2 0}$ \\
$45^{\circ}$ & 0.750 & 0.750 & 0.750 \\
$90^{\circ}$ & 0.750 & 0.719 & 0.813 \\
\hline
\end{tabular}

\subsection{AutoML with TPOT}

Using the same formalism, the classification scores of a classifier built by the TPOT algorithm are presented in Tab. 4 Scores are for the best model found. Due to the duration of a run (nearly 15 hours), we do not cross-validate each models thru the search process.

Table 4: AutoML classification scores.

\begin{tabular}{|c|c|c|c|}
\hline Angle & dataset_pola & dataset_nopola & dataset_bench \\
\hline 3 angles & 0.876 & 0.876 & $\mathbf{0 . 9 4 8}$ \\
$0^{\circ}$ & 0.820 & 0.875 & $\mathbf{0 . 9 2 3}$ \\
$45^{\circ}$ & 0.820 & 0.769 & 0.820 \\
$90^{\circ}$ & 0.846 & 0.820 & 0.889 \\
\hline
\end{tabular}

\section{DISCUSSION}

\subsection{Quality classification}

Good performances are achieved with at least $90 \%$ of correct classified parts in every case. With selected Machine Learning models, a maximum of $94.8 \%$ success rate is reached. This show that our vision based control method is meaningful on our case, even with a small dataset and in a variable environment. However, the results show that classification scores are not evolving very much with the different datasets and types of images. In the case of the hand-crafted features, the different extraction methods are not leading to the same results. In this case, the DMD features are better than Haralick. We limited ourselves to these two ones in this study, but would be interesting to try more types of features in the future to see if some of them are better to enhance the defect information.

This study is a first exploration and the proposed method needs to be evaluated on other types of parts, with multiple defects and different materials. With the Sacred framework, we will be able to quickly reproduce this study on a larger dataset. An industrial production of more than one thousand parts must be studied with our system to validate its performances and push further the analyse of polarization measurements. Finally, full high definition images could be used to better infer part quality.

\subsection{Polarimetry}

Even if overall good performances are reached, the benchmark dataset scores are always very close or even higher than the scores with polarization. Consequently, it is not possible at that stage to draw a conclusion about the interest of polarization to magnify visual defects in this specific case. To push further our study of polarization, we identify two main ways of improvement for our system. The first one is to verify if the polarization information is well captured by our sensor. As the sensor was not calibrated, we may not measure the real degree of linear polarization. The configuration of the lighting and the quality of the polarized filters we used may prevent us to get satisfying measurements. The second improvement is to implement a more complete pre-processing pipeline to enhance the polarization information before the machine learning process.

Finally, some defects are caused by deformations of surfaces and imply a polarization of the reflected light. Some other defects are caused by material transformation (burn, drip, freezing) and have a lesser impact on 


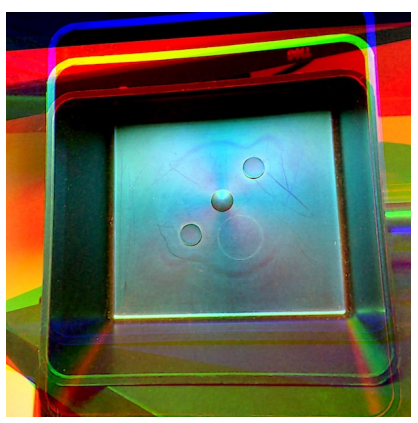

(a) $0^{\circ}, 45^{\circ}, 90^{\circ} \rightarrow$ BGR.

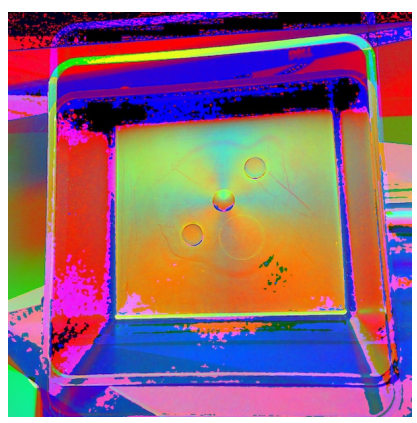

(b) Difference to $0^{\circ} \rightarrow$ BGR.

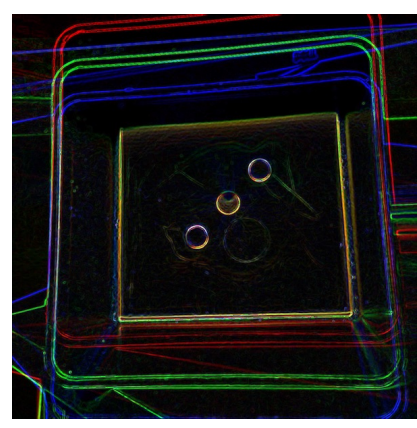

(c) Diff + Gaussian + Sobel.

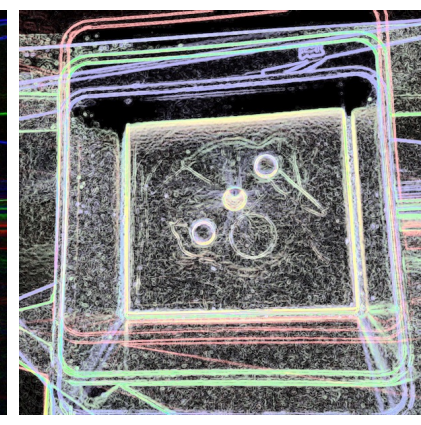

(d) Prev. with tuned hist.

Figure 3: Illustration of preprocessing methods.

reflected light polarization. The contribution of polarization for visual control should then be more or less important depending on the type of parts that are checked. Applying our method to other type of parts with various materials and defect, as introduced in the previous subsection, would then be also interesting to evaluate the interest of polarimetry. The next subsection is dealing with pre-processing ideas for the continuation of this study.

\subsection{Images pre-processing}

We have already begun to investigate different color mapping techniques to pre-process polarized images. The most basic method would be to use polarized images as a color mapping. The variation of the Degree Of Linear polarization (DOLP) maps with the color gradient. In Figure 3 (a), the red channel maps to the blue channel of the $90^{\circ}$ linear polarized camera, green to $45^{\circ}$ and blue to $0^{\circ}$. We choose to only use the blue channel and to not grayscale convert images to increase contrast on our specific problem. In Figure 3 (b), we propose a color mapping where the reference image is set to the $0^{\circ}$ polarized and the green and blue channels are respectively the difference between the $45^{\circ}$ and $0^{\circ}$ and between the $90^{\circ}$ and $0^{\circ}$ polarized images.

We did not find a lot of literature dealing with this problematic and with the evaluation of pre-processing methods on polarized images for defect enhancement. It is more common to find filtering methods applied to defects detection. In many applications, defects induce brutal contrast changes enabling the use of Laplacian filtering methods. ${ }^{29}$ Other methods include Gabor filtering, ${ }^{30}$ Wavelet transform ${ }^{31}$ or the Discrete Cosines Transform. An interesting overview on image processing for polarization is proposed in a recent survey by Mihoubi et al ${ }^{32}$ Authors evaluate different filtering methods with respect to the Peek Signal to Noise Ratio (PSNR). Additional works on defects detection with Convolutional Neural Networks use Gaussian filtering ${ }^{[3]}$ In Figure 3 (c) \& (d), we show the effect of a Sobel filter. The result is quite noisy. More advanced methods could be applied. In the present work, we do not evaluate the influence of filtering on the defect detection performances. This is an ongoing work.

\subsection{Optimal Machine Learning pipeline}

A quick comparison of the results for the different machine learning pipelines shows that Deep Learning give overall inferior results than more classical approaches on this specific use case, even with using Tranfer Learning. Extending the dataset with measuring more parts could help to get better results. Another way of improving could lie in a better pre-processing of the data as we proposed previously. Finally, a larger dataset could lead to achieve higher Deep Learning scores.

About the use of AutoML, the TPOT algorithm found the best solution after running half a day. To use or not AutoML depend on the answer: is it more costly to hand-craft a feature extraction pipeline or to run AutoML? Even on our case, a best solution was found in half a day and overall we spent more time designing our machine learning pipelines by ourselves. 


\section{CONCLUSION}

In this article we explore polarimetric imaging applied to visual quality control of plastic modeled parts. We present a new device for the acquisition of images with $0^{\circ}, 45^{\circ}$ and $90^{\circ}$ polarization angles. This device is intended to be used in industrial environment with acquisition variability. We discuss about the interest of polarization for visual quality control through the use of several machine learning algorithms. The classification task performed on the datasets show overall good performances (over $90 \%$ of successful classifications) despite a variable environment. The Discrete Modal Decomposition feature extraction and the TPOT AutoML methods get the best scores. However, the results did not allow us to conclude about the interest of polarization to magnify visual defects on this specific case.

To push further this study, we identify several perspectives. One consists in using different pre-processing methods to filter the data, but also to magnify the information coming from the polarization. To the same extend, we could also try to extract other types of hand-crafted features. Finally the dataset should be extended, especially for deep learning applications, and to run classification tasks on other types of parts with different shapes and materials.

\section{ACKNOWLEDGMENTS}

This work was funded by the french Banque Publique d'Investissement under the FUI SAPRISTI project, initiated by the $21^{\text {st }}$ call for projects of the Fonds Unique Interministériel. Molded parts used in the dataset were produced at the CT-IPC Technical Center.

\section{REFERENCES}

[1] Nagorny, P., Pillet, M., Pairel, E., Goff, R. L., Loureaux, J., Wali, M., and Kiener, P., "Quality prediction in injection molding," in [2017 IEEE International Conference on Computational Intelligence and Virtual Environments for Measurement Systems and Applications (CIVEMSA)], 141-146 (June 2017).

[2] Nagorny, P., Lacombe, T., Favreliere, H., Pillet, M., Pairel, E., Le Goff, R., Wali, M., Loureaux, J., and Kiener, P., "Generative adversarial networks for geometric surfaces prediction in injection molding: Performance analysis with discrete modal decomposition," in [2018 IEEE International Conference on Industrial Technology (ICIT)], 1514-1519, IEEE (2018).

[3] Marschner, S. R., Westin, S. H., Lafortune, E. P. F., and Torrance, K. E., "Image-based bidirectional reflectance distribution function measurement," Applied Optics 39, 2592-2600 (June 2000).

[4] Ghosh, A., Achutha, S., Heidrich, W., and O'Toole, M., "BRDF Acquisition with Basis Illumination," in [2007 IEEE 11th International Conference on Computer Vision], 1-8 (Oct. 2007).

[5] Denizart, O., Vincent, M., and Agassant, J. F., "Thermal stresses and strains in injection moulding: experiments and computations," Journal of Materials Science 30, 552-560 (Jan. 1995).

[6] Wolff, L. B. and Andreou, A. G., "Polarization camera sensors," Image and Vision Computing 13 (Aug. 1995).

[7] Bradski, G., "The OpenCV Library," Dr. Dobb's Journal of Software Tools 25(11), 120-125 (2000).

[8] Garrido-Jurado, S., Muñoz-Salinas, R., Madrid-Cuevas, F. J., and Marín-Jiménez, M. J., "Automatic generation and detection of highly reliable fiducial markers under occlusion," Pattern Recognition 47(6), 2280$2292(2014)$.

[9] Garrido-Jurado, S., Muñoz-Salinas, R., Madrid-Cuevas, F. J., and Medina-Carnicer, R., "Generation of fiducial marker dictionaries using Mixed Integer Linear Programming," Pattern Recognition 51, 481-491 (Mar. 2016).

[10] Romero-Ramirez, F. J., Muñoz-Salinas, R., and Medina-Carnicer, R., "Speeded up detection of squared fiducial markers," Image and Vision Computing 76, 38-47 (Aug. 2018).

[11] Stokes, G. G., "On the Composition and Resolution of Streams of Polarized Light from different Sources," Transactions of the Cambridge Philosophical Society 9, 399 (1851).

[12] Brehat, F. and Wyncke, B., [Représentation des états de polarisation des ondes lumineuses], Publibook, Paris (2003). 
[13] Haralick, R. M., Shanmugam, K., and Dinstein, I., "Textural Features for Image Classification," IEEE Transactions on Systems, Man, and Cybernetics SMC-3, 610-621 (Nov. 1973).

[14] Pentland, A. P., "Automatic extraction of deformable part models," International Journal of Computer Vision 4, 107-126 (Mar. 1990).

[15] Lacombe, T., Exploitation of a multilighting information for a generic approach of industrial automated visual quality control., PhD thesis, Communauté Université Grenoble Alpes (Sept. 2018).

[16] Masci, J., Meier, U., Ciresan, D., Schmidhuber, J., and Fricout, G., "Steel defect classification with MaxPooling Convolutional Neural Networks," in [The 2012 International Joint Conference on Neural Networks (IJCNN)], 1-6 (June 2012).

[17] Wen, S., Chen, Z., and Li, C., "Vision-Based Surface Inspection System for Bearing Rollers Using Convolutional Neural Networks," Applied Sciences 8, 2565 (Dec. 2018).

[18] Lecun, Y., Bottou, L., Bengio, Y., and Haffner, P., "Gradient-based learning applied to document recognition," Proceedings of the IEEE 86, 2278-2324 (Nov. 1998).

[19] Chollet, F. and others, "Keras," (2015).

[20] Kim, S., Kim, W., Noh, Y., and Park, F. C., "Transfer learning for automated optical inspection," in [2017 International Joint Conference on Neural Networks (IJCNN)], 2517-2524 (May 2017).

[21] Simonyan, K. and Zisserman, A., "Very Deep Convolutional Networks for Large-Scale Image Recognition," arXiv:1409.1556 [cs] (Sept. 2014).

[22] "Weakly Supervised Learning for Industrial Optical Inspection | Heidelberg Collaboratory for Image Processing (HCI)," (2007).

[23] Sandler, M., Howard, A., Zhu, M., Zhmoginov, A., and Chen, L.-C., "MobileNetV2: Inverted Residuals and Linear Bottlenecks," arXiv:1801.04381 [cs] (Jan. 2018).

[24] Olson, R. S., Urbanowicz, R. J., Andrews, P. C., Lavender, N. A., Kidd, L. C., and Moore, J. H., "Automating biomedical data science through tree-based pipeline optimization," arXiv:1601.07925 [cs] (Jan. 2016).

[25] Olson, R. S., Bartley, N., Urbanowicz, R. J., and Moore, J. H., "Evaluation of a Tree-based Pipeline Optimization Tool for Automating Data Science," in [Proceedings of the Genetic and Evolutionary Computation Conference 2016], GECCO '16, 485-492, ACM, New York, NY, USA (2016).

[26] Breiman, L., "Pasting Small Votes for Classification in Large Databases and On-Line," Machine Learning 36, 85-103 (July 1999).

[27] Greff, K., Klein, A., Chovanec, M., Hutter, F., and Schmidhuber, J., "The Sacred Infrastructure for Computational Research," in [Proceedings of the 15th Python in Science Conference], 49-56 (2017).

[28] Pedregosa, F., Varoquaux, G., Gramfort, A., Michel, V., Thirion, B., Grisel, O., Blondel, M., Prettenhofer, P., Weiss, R., Dubourg, V., Vanderplas, J., Passos, A., Cournapeau, D., Brucher, M., Perrot, M., and Duchesnay, E., "Scikit-learn: Machine learning in Python," Journal of Machine Learning Research 12, 2825-2830 (Nov. 2011).

[29] Yun, J. P., Choi, S., Seo, B., and Kim, S. W., "Real-time vision-based defect inspection for high-speed steel products," Optical Engineering 47, 1-8, 8 (July 2008).

[30] Kumar, A. and Pang, G. K. H., "Defect detection in textured materials using Gabor filters," IEEE Transactions on Industry Applications 38, 425-440 (Mar. 2002).

[31] Hu, M. C. and Tsai, I. S., "The Inspection of Fabric Defects by Using Wavelet Transform," The Journal of The Textile Institute 91, 420-433 (Jan. 2000).

[32] Mihoubi, S., Lapray, P.-J., and Bigu, L., "Survey of Demosaicking Methods for Polarization Filter Array Images," Sensors 18, 3688 (Nov. 2018).

[33] Tao, X., Zhang, D., Ma, W., Liu, X., and Xu, D., "Automatic Metallic Surface Defect Detection and Recognition with Convolutional Neural Networks," Applied Sciences 8, 1575 (Sept. 2018). 